\title{
Anabases
}

ANABASES Traditions et réceptions de l'Antiquité

$23 \mid 2016$

Varia

\section{Un magistrat passionné d'étruscologie : Charles Casati (1833-1919)}

Dominique Briquel

\section{(2) OpenEdition}

\section{Journals}

Édition électronique

URL : http://journals.openedition.org/anabases/5549

DOI : 10.4000/anabases.5549

ISSN : 2256-9421

Éditeur

E.R.A.S.M.E.

\section{Édition imprimée}

Date de publication : 2 mai 2016

Pagination : $27-43$

ISSN : 1774-4296

\section{Référence électronique}

Dominique Briquel, «Un magistrat passionné d'étruscologie : Charles Casati (1833-1919) », Anabases

[En ligne], 23 | 2016, mis en ligne le 02 mai 2019, consulté le 21 octobre 2019. URL : http:// journals.openedition.org/anabases/5549; DOI : 10.4000/anabases.5549

(c) Anabases 


\title{
Un magistrat passionné d'étruscologie: Charles Casati (1833-1919)
}

Dominique BRIQUEL

\begin{abstract}
- n 1909, un des représentants les plus éminents des études étrusques était le — Suédois Olof August Danielsson (I852-Ig33), qui était professeur à Uppsala ' — Il fut associé dès I883 à l'étruscologue allemand Carl Pauli (I839-I9or) qui était alors en train de lancer le projet d'un Corpus Inscriptionum Etruscarum ${ }^{2}$, pendant dans ce secteur du Corpus Inscriptionum Latinarum. Cette entreprise visait à fournir un répertoire complet des inscriptions étrusques, fondé sur le principe de l'autopsie, ce qui n'avait pas été le cas du Corpus Inscriptionum Italicarum de l'Italien Ariodante Fabretti, publié à Turin en i867. Danielsson joua un rôle central dans l'entreprise, si bien que, lorsque Pauli mourut en Igor, ce fut naturellement lui qui présida en 1902 à la publication du premier volume, consacré aux inscriptions du Nord de la Toscane. Mais, si Pauli et Danielsson s'étaient rendus à maintes reprises en Italie, y étudiant méticuleusement tous les documents, ils avaient omis d'étendre leur enquête à d'autres pays, comme la France ou la Grande-Bretagne alors que, dès cette époque le Louvre et le British Museum possédaient de riches fonds épigraphiques étrusques. Ce n'est qu'en igog que Danielsson put remédier à cette carence: il se rendit alors à Paris, puis à Londres pour y examiner les documents étrusques - dont certains auraient dû figurer dans le volume I du CIE, ou y étaient donnés à partir de publications anciennes avec des lectures erronées. Mais le travail que Danielsson effectua alors demeura inconnu puisque les notes qu'il prit furent consignées dans un journal de voyage, un Tagebuch rédigé en allemand,
\end{abstract}

1 Sur la personnalité et la carrière de Danielsson, Wikander 2003, p. II-27.

2 L'entreprise, fondée comme celle du CIL sur la base de la distribution géographique des documents, est toujours en cours. 
déposé à la bibliothèque de l'université d'Uppsala, dans un fonds d'archives dont l'existence resta ignorée jusqu'en $2003^{3}$.

L'intérêt de ce journal est considérable. Nous avons eu l'occasion de l'évoquer pour ce qui est des inscriptions du Louvre dans une communication à l'Académie des inscriptions et belles-lettres ${ }^{4}$. Mais le Tagebuch jette une lumière sur un personnage assez oublié de nos jours, un ancien élève de l'École des Chartes devenu magistrat qui fut, à défaut d'être un véritable savant, un amateur d'antiquités étrusques passionné, Charles Casati, ou, comme il se faisait appeler, Charles Casati de Casatis (I833-rgrg). Au cours de son passage à Paris, Danielsson examina en effet la petite collection épigraphique que possédait ce personnage, qui l'avait invité à voir les inscriptions qu'il possédait.

On peut donc, d'après les notes de Danielsson, se faire une idée exacte de ces documents, dont plusieurs n'avaient jamais été signalés. Il s'agit de:

- un premier cippe rond, en forme de colonne, portant à son sommet une inscription donnant le nom du défunt. C'est un type de monument bien connu dans les nécropoles d'Orvieto. Celui-ci avait été vu par Danielsson au domicile parisien de Casati le jjuin rgog. L'inscription est śêres murcnas, ce qui renvoie à un homme dont le prénom est Śêre et le gentilice Murcna, indiqués ici au génitif. Danielson y avait reconnu un document déjà connu, $C I E 5040^{5}$. Découvert en janvier I885 au cours de fouilles menées par l'ingénieur Riccardo Mancini dans la nécropole de la Cannicella à Orvieto, l'antique Volsinies ${ }^{6}$, il fut publié dans les Notizie degli Scavi de $1886^{7}$. Casati lui-même en fit état lors d'une de ses interventions à l'Académie des inscriptions et belles-lettres, le 5 février I886, où il apporta en pleine séance ce cippe, interprétant le texte qu'il portait comme une formule onomastique au génitif, avec un prénom qu'il lisait Setre (sans relever que le premier signe était non la sifflante simple $\mathrm{S}$, mais la sifflante marquée, notée par un signe de forme $\mathrm{M}$, ni que le mot comportait non un $\mathrm{T}$, mais un thêta) et un gentilice qu'il rendait par le latin Murcanius (alors que Murcius aurait été plus exact) ${ }^{8}$. Dans la notice du CIE parue en rgo2, Pauli et Danielsson, ignorant que l'objet avait été acquis par Casati, estimaient son sort ultérieur inconnu («nunc ubi adservetur, non comperimus»);

3 Cette découverte est due à Örjan Wikander et son épouse Charlotte, aujourd'hui disparue. Voir Wikander 2003.

4 Briquel 2013.

5 On lit dans le journal à la date du 7 juin rgog: «Bei diesem ersten Besuche machte ich einen Ablklatsch des Orvietaner cippus cIE.5o40 śeAresmurcnas. Höhe o,25 (o,24 Manc. schien mir zu niedrig x Diam. o,I7 = Manc.) »

6 Sur les fouilles menées par Mancini à Orvieto, Bonamici I994, en part. p. 7-9.

7 Mancini I885, p. I6-I7.

8 Voir Casati ı886, p. I40 (ne notant ni la sifflante forte initiale ni l'aspirée du prénom, rendu par lui comme Setre). 
- deux autres cippes analogues, de même provenance, étaient entrés dans la collection Casati. Ceux-ci (de même que le cippe suivant, de Tarquinia) étaient conservés dans la propriété qu'il possédait dans le Loiret, sur la commune de Boiscommun, La Javelière 9 . Mais Danielsson avait eu l'idée de montrer à un membre du personnel de Casati comment faire des estampages, qu'il étudia lors de sa dernière visite au collectionneur, le 3ojuin $\operatorname{rg} 9^{10}$. Le premier de ces cippes portait l'inscription

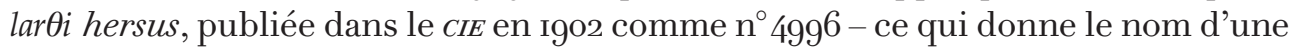

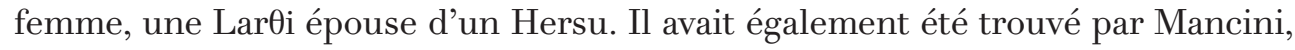
cette fois au cours de l'hiver I874-1875 dans la nécropole du Crocifisso del Tufo ${ }^{11}$, et avait été ensuite déposé par lui à l'Opera del Duomo à Orvieto ${ }^{12}$. Mais lorsque, dans le cadre de la préparation du CIE, Pauli et Danielsson se rendirent à Orvieto, en I885-ı886, ils n'y virent pas cette pièce, et elle ne s'y trouvait pas davantage en I902, quand l'Italien Bartolomeo Nogara, qu'ils s'étaient alors adjoint comme collaborateur $^{13}$, y retourna ${ }^{14}$ - ce qui suppose que Mancini l'ait retirée du bâtiment de l'Opera del Duomo et en ait disposé à sa guise ;

- le troisième cippe de type volsinien, également conservé à La Javelière et dont Danielsson avait fait faire un estampage ${ }^{15}$, était lui aussi déjà connu. Il s'agissait

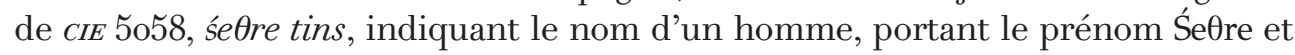
le gentilice Tins. Ce cippe avait été trouvé en ı880 à la Cannicella, toujours par Mancini ${ }^{16}$. Comme pour les deux autres cippes d'Orvieto, le devenir de celui-ci était inconnu (on lit dans le CIE: postea quo devenerit ignoratur);

9 Cette propriété, vendue en I92I après la mort de Casati, existe encore. Nous tenons à exprimer notre reconnaissance au propriétaire actuel, M. Patrick Masure, pour l'obligeance avec laquelle il nous a fourni des informations sur le devenir ultérieur du manoir.

10 On lit dans le Tagebuch à cette date : “Herr Casati auf seinem Besitztum "La Jave(l)lière” (bei Boiscommun, Loiret) Abklatsche, die ich bei meinem Abschiedsbesuche bei ihm 3o/6 og erhielt [eigentl(ich) von seinem Bedienter gemacht den ich instruirt und mit Papier versehen hatte] ". Ce cippe figure en première place des trois documents dont Danielsson fit faire des estampages : «a) Cippus, Basalt, Orvieto : o,345 x 0,I7 Diam. lar0i hersus cIE. 4996 (Masse auf der Rückseite der Ablklatsche). »

11 Körte ı877, p. Iเ6, n³; également Deecke ı877, p. IоI, $\mathrm{n}^{\circ} \mathrm{V}$.

12 Voir Gamurrini I88o, n⿳588. Sur l'Opera del Duomo d’Orvieto, dont les origines remontent au Moyen Âge, Lazzarini i994.

13 Wikander 2003, p.22-25.

14 On lit dans la notice du CIE : in museo Urbevetano a. I885.I886 non viderunt $P_{A}, D_{A}$ nec a. Igo2 reperire potuit NoG.

15 La description de l'objet dans le journal, qui suit celle de CIE 4996, est: «b) wie vorherg. o,26 h. x 0,I5 Diam. śeAre tins cIE 5o58. »

16 Mancini I88I, p.348, Helbig I88I. 
- le quatrième cippe de la collection, également déposé à La Javelière, était lui aussi resté inconnu dans la littérature épigraphique. Il était d'un type différent. Dans une lettre du i4août Igo8 à Danielsson, évoquée dans le Tagebuch, Casati avait défini cette pièce comme un "fragment de stèle » et avait donné un dessin peu satisfaisant des trois lignes de l'inscription ${ }^{17}$. Celle-ci était inscrite à l'intérieur d'un cadre rectangulaire. Cela correspond au type connu pour Tarquinia, où les cippes sont formés d'une colonne ou d'un pilier se dressant sur une base rectangulaire portant, sur sa face avant, le nom du défunt ${ }^{18}$. Ce point n'avait pas échappé à Danielsson, qui avait reconnu dans cet objet une base de cippe de “Corneto »c'est-à-dire de Tarquinia, qui ne reprit son nom ancien qu'en $1922^{19}$. Il avait également donné la lecture qui s'imposait du texte: la première ligne était occupée par le mot cnevna, nom de la famille étrusque Cneuna/Cnevna; la deuxième comportait le prénom ŚeӨre, avec une inversion du gentilice et du prénom qu'on rencontre parfois, spécialement à Tarquinia; la troisième ligne, avec Satna à compléter en Satnal, indiquait le nom de la mère, mis au génitif, en sous-entendant le mot "fils » ou “fille» - selon un usage, typique du monde étrusque, de référer un individu à son lignage maternel: la mère de ce Śêre Cnevna était une Satnei, membre féminin de la famille Satna;

- la collection comprenait aussi deux autres pièces inscrites, deux petites urnes en terre cuite, portant sur leur face avant un décor estampé et pourvues d'un couvercle représentant le défunt couché sur un lit. Ce type de réceptacle funéraire fut fabriqué en série à Chiusi aux $\mathrm{II}^{\mathrm{e}}$ et $\mathrm{I}^{\mathrm{er}}$ siècles av. J.-C., avec un décor estampé porté sur la face avant ${ }^{20}$. Il n'est donc pas étonnant que Casati en eût possédé deux. La première, de petite taille, avait sa façade ornée d'une scène d'adieux devant la porte des Enfers ${ }^{21}$. L'inscription, peinte sur le bord du couvercle, dont Danielsson

17 Pour la première ligne, Casati donnait, après une zone présentée comme «non lisible », une séquence evna: l'estampage montre qu'il s'agissait de cnevna, c'est-à-dire du gentilice Cnevna; pour la deuxième, il avait reconnu le prénom Śêre ; pour la troisième, son dessin commençait par noter la présence d'une « lettre douteuse », puis portait les quatre lettres avna; mais ce qu'il interprétait comme un $[\mathrm{v}]$ de forme $\mathrm{F}$ est en réalité un T et le mot se lit śatna.

18 Kaimio 20io.

19 On lit dans le Tagebuch, en date du 3o juin rgog: “c) "Stèle" in einer calcaire-artig Gestein, wahrscheinlich nenfro, nicht in Orvieto erworben, aber wo Herr Casati nicht mehr errinert sich. Nach dem Aussehen des Abklatsches würde ich sagen: "Cippus basis v. Corneto". Masse auf der Rückseite der Abklatsche vermesst 0,26 x 0,2I cnevna / śeAre/satna(l) al?»

20 Sclafani 20I0. Le Louvre possède 49 exemplaires de ces urnes, voir Briguet 2002, p. 40 - 127 .

21 Le Louvre possède cinq urnes de ce modèle (Briguet 2002, p. 99-IO4). 
estimait qu'il était non pertinent, donnait le nom d'un V(e)l Tutna Spaspu, homme qui portait le prénom Vel, le gentilice Tutna et le cognomen Spaspu ${ }^{22}$, cette formule de base étant complétée par Ar[n $\theta a l]$, prénom de son père au génitif, ce qui indique qu'il était fils d'un Arn $\theta$. Cette inscription, non répertoriée dans le cIE, n'aurait cependant pas dû rester inconnue: Casati l'avait présentée l'Académie le 2I septembre I888, avec il est vrai une lecture fautive vel. tutna spastu;

- Casati possédait une dernière inscription, encore une fois sur une urne en terre cuite de Chiusi, avec sur la face avant la scène dite du héros à l'araire, d'interprétation controversée, où on voit un homme torse nu brandissant son instrument contre une troupe de guerriers armés ${ }^{23}$. Danielsson l'étudia le i6juin ıgog, à la suite de l'urne précédente ${ }^{24}$. Le texte était vel: laucina: ataluniaś, ce qui renvoie à un homme, Vel Laucina, fils d'une Atalunia.

Nous avons jusqu'à présent parlé de la collection ${ }^{25}$. Venons-en au collectionneur. Il ne s'était pas contenté de rassembler des pièces d'intérêt épigraphique, mais, voulant faire œuvre de savant, en avait présenté deux lors de séances de l'Académie. Car, même si Charles Casati n'exerça pas de fonction académique, on se trouve en présence d'un érudit et il s'était assuré une formation de base qui aurait pu déboucher sur une carrière scientifique. Il fut élève de l’École des Chartes avant de s'orienter vers le droit ${ }^{26}$.

22 Sur le cognomen, élément facultatif du système onomastique étrusque, Rix ig63.

23 Sur ces urnes, Briguet 2002, p.66-82. Le musée du Louvre possède treize urnes de ce type.

24 On lit dans le Tagebuch: «2. Ossuar v. Terrac. chius. Form o,35 l. x o,2I h. Pflugkampferrelief. Darauf Deckel mit eingehüllt liegender Figur, o,40 1. o,40 lang allzu für das Oss. zu gross. Inschrift ober. Vorderrand der Arca (Urne) rot gemalt. vel (I?) lau(2)cina:(3) atalu(4)n(5)iaś:(6) I) Zweifelhaft, ob Spur des Unterpunktes ganz unten. 2) Das Aussehen von $n$ und $c$ wird zum grossen Teil dadurch bestimmt, dass hier der Pflugschar in den Inschriftrand hereinragt. 3) Konnte nicht mehr als den Unterpunkt sehen. Möglicherweise oben Schatten des anderen. 4) Das $n$ sicher; auch vom linken Bein sieht man einen Schatten. 5) Die 2 Überreste von Vertikalhasten können wohl nur einem $n$ angehört haben. 6) Oben Spur eines Oberpunktes; unten alles abgestraft. Der Rest der Inschriftenrandes (m/ o,I2) völlig abgewichen - d(as) h(eisst) der Farbenunterzug des Grundes verschwunden, so dass der nackte Ton zu Tage tritt. »

25 Aucune de ces six pièces n'a été signalée depuis. Casati, resté célibataire, étant mort sans descendance, sa propriété de La Javelière fut léguée à sa gouvernante, MarieLouise Van der Meere, qui vendit le mobilier puis le château en octobre ig2i. Il n'existe malheureusement pas de catalogue des pièces mises en vente. Nous remercions P. Masure pour ces renseignements.

26 Notice sur Casati sur le site de l'École des Chartes http://catalogue.enc.sorbonne.fr/ expositions/fonds-casati/biographie-de-m-casati. 
Ce fut un personnage assez singulier. Né à Lyon dans une famille d'origine italienne, il n'hésita pas à s'engager dans l'armée lors de la campagne d'Italie en I859 et, à côté d'une carrière très honorable de magistrat, il mena une activité intellectuelle intense, dans les domaines les plus divers. Véritable polygraphe, il exprima ses idées sur la politique internationale, à propos de l'Italie (Rome ou Florence, quelle doit être la capitale de l'Italie?, I86i, La Meilleure Alliée de la France, c'est l'Italie, I864, Venise et les traités de I866, I866), l'empire ottoman (Recueil de la question d'Orient, I86o) ou les affaires scandinaves (La Monarchie scandinave. À propos de la question danoise, I865), il s'intéressa à l'histoire de l'art (L'Art français primitif, I905), étudia des archives (Villes et châteaux de la vieille France, Duché d'Auvergne, d'après les manuscrits du chanoine Audigier et du hérault d'armes Revel, I9oo) et émit des idées en matière de législation (Projet de loi sur la propriété littéraire et artistique, $\mathrm{I} 880$ ).

Mais il eut une prédilection particulière pour le domaine étrusque. Le dernier ouvrage qu'il fit paraître, chez Auguste Picard à Paris en i9ı4, fut Les Étrusques, leur langue et leur civilisation. Et c'est en priorité sur l'étruscologie que portèrent ses très nombreuses interventions, faites sous des formes diverses (hommages de travaux, envoi de lettres ou de documents, lectures de mémoires) à l'Académie des inscriptions et belles-lettres, dont on trouve la trace dans les comptes rendus des séances entre 1873 et I9oI $^{27}$. Nous les énumérons ci-dessous :

- I8 novembre I873 (CRAI, p.436): hommage à l'Académie présenté par Alfred Maury de son opuscule La lettre $\Lambda$ dans l'alphabet étrusque.

- 9 mai I879 (CRAI, p. IO3): remise à l'Académie, par l'intermédiaire du linguiste Michel Bréal, d'un estampage d'une inscription étrusque, avec interprétation de la première ligne par Casati.

- 5 décembre ı879 (CRAI, p.493): hommage à l'Académie, présenté par Eugène de Rozière, de son article «Sarcophage étrusque de Chiusi » paru dans la Gazette archéologique de 1879 , p. I38-ı64.

- 25 juin I88o (CRAI, p.I34): remise à l'Académie, par l'intermédiaire de Michel Bréal, de l'“estampage de deux inscriptions étrusques récemment découvertes à Tarquinia.»

- 20 octobre I882 (CRAI, p. 268): «M. Casati lit un mémoire sur l'État actuel de la science historique touchant les Étrusques. »

27 Casati fit partie à partir de 1873 de la Société nationale des antiquaires de France. Mais les quelques interventions qu'il y fit ne concernèrent qu'exceptionnellement les antiquités étrusques. Le Bulletin évoque rapidement deux interventions de sa part dans ce domaine, en I879 sur les nécropoles étrusques (p. I58) et en i886 sur la découverte d'une tombe étrusque à Colonna près de Grosseto (p.207). Visiblement, il considérait que son activité dans ce secteur regardait en priorité l'Institut. 
- 5 octobre i883 (CRAI, p.394-395): lecture d'une «lettre relative à la découverte d'une tombe étrusque aux environs d'Orvieto. »

- 2 mai ı884 (CRAI, p. I89): “M. Casati donne lecture d'un mémoire sur La civilisation étrusque d'après les monuments.» Le texte figure p.238-243, où il est suivi d'une autre étude, “Les noms de famille étrusques et les inscriptions bilingues », p. $243-248$.

- I8 juillet I884 (CRAI, p.408): remise à l'Académie du texte de l'étude lue le 2 mai.

- 2I novembre i884 (CRAI, p.504): “deuxième étude lue devant l'Académie, Fortis Etruria, la civilisation étrusque d'après les monuments. »

- I7 avril I885 (CRAI, p. 93): “M. Casati commence une lecture sur l'Épigraphie de la numismatique étrusque.»

- 24 avril I885 (CRAI, p.94): “M. Casati continue et achève la lecture de son mémoire. »

- I7 juillet I885 (CRAI, p. 225): “M. Casati lit une notice sur Les marques de valeur que portent les monnaies étrusques. »

- 5 février ı886 (CRAI, p. I5): “M. Casati fait une communication au sujet de quelques débris trouvés dans les tombes étrusques et en explique l'origine et la destination. » C'est à cette occasion qu'il apporte le cippe CIE 5o40 et le commente.

- Io septembre ı886 (CRAI, p.364): “M. Casati lit une étude sur "les caractères de la Gens Romana et l'origine des noms de famille chez les Étrusques". »

$-\mathrm{I}^{\mathrm{er}}$ octobre $\mathrm{I} 886$ (CRAI, p. 425) : “M. Casati continue la lecture de son mémoire: Les origines étrusques de la gens romaine. »

- 4 mars I887 (CRAI, p. I39-I40): “M. de Rozière fait hommage à l'Académie (de) Mémoires de l'Académie étrusque (section française): La Gens. Origine étrusque de la gens romaine, par M.C.-Ch. Casati (Paris, I887). »

- 23 septembre I887 (CRAI, p.33o-33I) : “M. Casati commence une communication sur les sarcophages étrusques. »

- 9 décembre 1887 (CRAI, p. I5): dépôt à l'Académie de Fortis Etruria. Origines étrusques du droit romain, Paris, I888.

- I8 mai ı888 (CRAI, p. I8I, 204-206): lecture d'une lettre adressée de Florence en date du i6mai i888 au président de l'Académie sur des tombes découvertes à Orvieto.

- 2I septembre I888 (CRAI, p.40I-402) : “lecture sur les musées étrusques récemment formés en Italie, et sur les œuvres d'art qu'ils renferment. » C'est lors de cette séance que Casati montra aux membres de l'académie une de ses deux urnes inscrites.

- I3 septembre I889 (CRAI, p.298-299): “M. Casati fait une nouvelle communication sur l'archéologie étrusque.» Il fait passer "plusieurs spécimens qui proviennent des dernières fouilles faites à Orvieto, à Chiusi et à Pérouse », des bijoux d'or, des objets en bronze, deux exemples d'aes rude d'Orvieto et deux pièces d'argent de Populonia. 
- 7 novembre i89o (CRAI, p.424): présentation de reproductions des peintures de la tombe des Hescanas d'Orvieto (appelée par lui tombe de la famille Thescanas). À cette occasion “M. Casati fait passer sous les yeux des membres de l'Académie divers bijoux de bronze, provenant d'Orvieto, puis un miroir ».

- 24 juillet I89I (CRAI, p.26I-262): communication sur l'origine du lion de SaintMarc à Venise, considéré comme étrusque.

- 3 février I8g3 (CRAI, p. II): note complémentaire sur le lion de Saint-Marc à Venise, où «M. Casati maintient les conclusions du travail qu'il a lu devant l'Académie à ce sujet».

- IO mars I893 (CRAI, p.57-58): “M. Casati adresse à l’Académie le mémoire de M. Giacomo Boni, intitulé: Il leone di San Marco (bronzo veneziano del milleduecento), Rome, I892, avec une lettre dans laquelle il combat les conclusions de ce travail. »

- 3 novembre I8g3 (CRAI, p.375-376): «M. Casati fait une communication sur un texte de droit étrusque»; il s'agit de la prophétie de Vegoia, conservée par les Gromatici Veteres, et «l'étude dont il donne lecture est un fragment du livre intitulé Jus antiquum qu'il va publier ».

- 8 juin I894 (CRAI, p.244) : remise à l'Académie, par l'intermédiaire de M. Bréal, de Jus antiquum, Paris-Milan, I894.

- 4 janvier Igor (CRAI, p.36): remise à l'Académie, par l'intermédiaire de M. Bréal, de la brochure Numismatique étrusque. Quel mode de classement doit-on adopter?, extrait des Mémoires du congrès international de numismatique de Igoo.

Casati multiplia donc les interventions à l'Académie sur les domaines les plus divers de l'étruscologie, archéologie, histoire de l'art, linguistique, droit, muséographie. Il tint visiblement à se poser comme le grand spécialiste de la matière, avec une ostentation qui l'amenait à se présenter comme représentant la section française de l'Académie étrusque, à faire passer dans la salle de séances des pièces de sa collection, ou à souligner, dans sa lettre du ı6 mai ı888, qu'il était un personnage assez important pour que le fouilleur d'Orvieto, Mancini, lui fît l'honneur d'ouvrir en sa présence une tombe non encore fouillée. Malgré tous ses efforts, il n'eut pas la satisfaction d'être élu correspondant de l'Académie, poste pour lequel il avait affiché ses ambitions dès $1874^{28}$. Mais il faut reconnaître que, par ses

28 On lit dans les CRAI pour la séance du 26 novembre i874, p. 294-295: «M. Casati se met sur les rangs pour l'une des places de correspondant de l'Académie actuellement vacantes. Sa lettre est renvoyée à la Commission qui sera chargée de dresser la liste des candidats. » 
relations avec l'Italie, et notamment avec Mancini ${ }^{29}$, il joua un rôle non négligeable dans ce que nous appellerions aujourd'hui la veille scientifique et la diffusion des nouveautés importantes dans le secteur des antiquités étrusques.

La notice biographique qu'on lit sur le site de l'École des Chartes évoque la passion qui animait Casati envers ces antiquités étrusques. C'est bien l'impression que donnent par exemple ses dernières interventions importantes à l'Académie, où il défendit avec acharnement son idée de l'origine étrusque du lion ailé de la piazzetta San Marco à Venise, se lançant dans une polémique avec le fouilleur du Forum, G.Boni, qui avait eu l'outrecuidance de soutenir que c'était une œuvre médiévale. Mais il paraît difficile de faire de lui un véritable savant. Pour nous en tenir aux points d'épigraphie et de langue, nous avons vu que ses lectures n'étaient pas très sûres, y compris lorsqu'il s'agissait des documents qu'il avait en sa possession. Il connaissait le CII de Fabretti, sur lequel il ne tarissait pas d'éloges dans son ouvrage sur la langue de $194^{30}$; il n'empêche qu'il n'en tint pas compte lorsque, dans son exposé sur «Les noms de famille étrusques et les inscriptions bilingues », paru dans les CRAI de I884, il n'hésitait pas à présenter comme inédite une inscription portée sur une pointe de lance en bronze, qu'il avait vue au Louvre, alors que celle-ci, achetée par le musée en ı864 à Alessandro Castellani, figurait dans le recueil de Fabretti sous le $n^{\circ} 2095$ ter $\mathrm{c}^{31}$.

Sur un plan plus général, son approche de la langue n’apparaît pas très sérieuse. Dans son travail de I9ı4, il s'insurgeait contre ceux qui affirmaient que nous ne connaissons que très peu de mots étrusques: “Nous serions vraiment bien à plaindre si nous n'avions comme point de repère pour connaître la langue étrusque que deux ou quelques mots, tandis qu'il y a quatre mille mots (que) nous connaissons ${ }^{32}$. " Mais, à lire l'ouvrage, on s'aperçoit que ces quatre mille mots étaient avant tout les prénoms et noms de famille dont il donnait les équivalents latins, les noms mythologiques, empruntés au grec, les toponymes connus également

29 Ses rapports avec Mancini ne furent pas d'ordre seulement scientifique. Il est probable que c'est Mancini qui vendit à Casati les cippes de sa collection qui provenaient d'Orvieto. Tous les trois étaient issus de fouilles de l'ingegnere et s'il avait déposé celui portant l'inscription CIE 4996 à l'Opera del Duomo où Pauli, Danielsson, Nogara le cherchèrent en vain, il avait dû le reprendre par la suite. Parfois présenté comme un savant désintéressé (Klakowicz I974, p.66), Mancini fut en réalité un acteur important du commerce des antiquités à son époque (Bonamici I994, en part. p.5, n. I9).

30 Casati IgI4a, p. VII : «le glossaire le plus complet et le mieux fait est celui de mon ancien et regretté ami le sénateur Ariodante Fabretti, Glossarium Italicum (trois mille pages in-folio) et le Corpus inscriptionum italicarum antiquioris aevi, en trois volumes in folio. »

31 Inscription CIE IO86I, ET, Vs 4.37.

32 Casati igía, p. VI. 
sous leur forme latine ou grecque. Il est évident qu'un linguiste ne se fonderait pas sur ce type de vocabulaire pour définir une langue. Mais Casati n’était en rien un linguiste. On le voit même lorsqu'il avançait des remarques justifiées sur le sens des documents qu'il citait: ainsi il avait reconnu, dans les inscriptions funéraires étrusques, dans les terminaisons en -al des métronymes une référence au nom de la mère du défunt, dans celles en $-s a$ du gamonyme qui pouvait accompagner la formule onomastique des femmes une référence à leur mari ${ }^{33}$. Mais il présentait ces éléments $-a l$ et $-s a$ comme des suffixes spécialisés, ayant ce seul sens : or une analyse vraiment grammaticale, tenant compte des autres occurrences de ces éléments, montre que - al est une terminaison de génitif et -sa un pronom enclitique signifiant “celui de», dont l'emploi onomastique n'est qu'un cas particulier de la fonction dans la langue.

On rencontre parfois des affirmations totalement fausses. L'une d'elles concerne l'inscription prétendument inédite sur pointe de lance qu'il avait évoquée à l'Académie le 2 mai ı884. Il en donnait une lecture correcte, Arth. Cecna. Suthina. Mais il l'interprétait comme «Aruns: Cecina, Saluti ou pro salute.» Il donnait donc au dernier mot, śufina, le sens de “à la déesse Santé» ou “pour sa santé ${ }^{34}$ ». Or le sens de ce mot ne souffre aucune contestation: dérivé au moyen d'un suffixe de dérivation -na du mot śu $\theta i$ qui veut dire «tombe» et signifiant donc “tombal», il apparaît porté sur de nombreux objets appartenant au mobilier funéraire d'une tombe, servant à les désigner comme faisant partie des biens remis au défunt pour l'accompagner dans l'au-delà ${ }^{35}$. Casati en était resté à une interprétation qui avait été celle de Lanzi en I789 et figurait encore dans le Glossarium Italicum qui complétait le $c I I$ de Fabretti en $1867^{36}$, mais n’était plus acceptable en i884. Or Casati connaissait l'explication de ce mot à partir du nom de la tombe: il en faisait état dans sa communication. Mais c'était pour la refuser: “Cette inscription peut servir à combattre l'interprétation donnée récemment du mot suthi, "sépulcre », interprétation qui pourrait s'expliquer sur un sépulcre ou un vase funéraire, mais non sur un fer de lance. »

Pourquoi Casati était-il aussi négatif à l'égard de ce qui est l'interprétation juste du terme? Il faut faire intervenir l'idée qu'il se faisait de la langue étrusque. Comme il devait encore l'affirmer dans son opuscule de igi4, à une époque où cette conception était complètement dépassée, il fallait selon lui «reconnaître dans la langue

33 Casati ıgı́a, p. IX : «La terminaison al indique le nom de la mère et la terminaison $s a$ le nom du mari. »

34 Voir CRAI, I884, p. 245.

35 Fontaine 1995 .

36 Lanzi I789, II, p.48I-2; voir A. Fabretti, Glossarium Italicum, p. I723, avec d'autres références. 
étrusque une langue indo-européenne, langue sœur des langues grecques et latine à tel point que l'on peut hésiter souvent pour savoir si l'inscription funéraire est une inscription latine ou étrusque ${ }^{37}$ ». C'est cela qui justifiait l'interprétation de

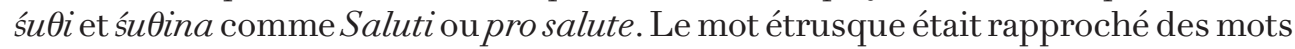
grecs $\sigma \tilde{\omega} \varsigma, \sigma \omega \tau \dot{\rho} \rho \iota \nu, \sigma \omega \tau \varepsilon \tilde{\imath} \rho \alpha$. Une telle méthode ne pouvait aboutir qu'à des résultats désastreux, y compris lorsque certaines bases de raisonnement étaient justes. Ainsi Casati avait relevé que les indications de l'âge du défunt dans les inscriptions funéraires (indications qu'on pouvait facilement repérer grâce à la présence d'un chiffre) étaient accompagnées des mots avil ou ril. De là la conclusion, fondée, que l'un des deux termes était le nom de l'année, l'autre une sorte d'adjectif au sens de “âgé de », correspondant au natus qu'on trouve dans des inscriptions latines. Mais, alors que c'est avil qui veut dire année, ril ayant le sens de natus, Casati défendait la solution inverse : avil était expliqué à partir des mots latins aevum, aevilis et recevait le sens de «âgé », ce qui faisait que le nom de l'année était ril ${ }^{38}$.

Il ne sert à rien de faire un bêtisier et d'énumérer toutes les erreurs qu'on trouve chez notre auteur. Nous nous bornerons à en citer une, qui renvoie à son premier travail sur l'étrusque ${ }^{39}$, où il étudia la valeur d'une lettre qu'on rencontre seulement dans quelques inscriptions étrusques récentes, surtout dans la région de Cortone, et qui a la forme du lambda majuscule grec, mais est une forme particulière de $\mathrm{M}$ que se sont donnée, à un certain moment, les scribes cortonéens. Il y défendait l'idée que le signe avait la valeur du $\Lambda$ grec, donc celle de [l]. Une des inscriptions sur lesquelles il s'appuyait était celle d'un document mis au jour en I840, le «lampadaire de Cortone», splendide lampe en bronze sur laquelle avait été fixée une petite plaque de bronze inscrite ${ }^{40}$. Or le second mot de l'inscription est un nom propre, qu'il faut lire Muśni; mais la graphie en est, si on reproduit la forme des lettres, $\Lambda$ VMNI, la première lettre étant cette forme particulière de $\mathrm{M}$ et la troisième le signe en forme de $\mathrm{M}$ qui, en étrusque, n'a pas la valeur de $[\mathrm{m}]$, mais celle de la sifflante marquée [ś]. Casati lisait en revanche lumni et voyait dans ce mot, porté sur un lampadaire, l'équivalent du latin lumen.Lumni aurait été le nom de la lumière, et donc de la lampe en étrusque.

Il défendait encore cette lecture erronée, et partant cette interprétation, en I9I $4^{41}$. À vrai dire, il l'avait trouvée chez Fabretti, qui, en I867, dans son Glossarium

37 Casati ı9ı́a, p. XV-XVI; pour les inscriptions funéraires, l'auteur se réfère à des épitaphes dans lesquelles on ne trouve que des noms propres, pour lesquels l'équivalence entre les formes étrusques et leurs correspondants latins ne pose aucun problème, mais ne prouve rien quant à la langue.

38 Casati igI4a, p. XVI-XVII.

39 Casati 1873.

40 Inscription CIE 443, ET, Co 3.I.

41 Casati igi/4b, p. XII. 
Italicum, n'avait pas encore reconnu la valeur de $[\mathrm{m}] \mathrm{du}$ “ $\mathrm{M}$ cortonese» et le lisait comme un lambda grec $^{\mathbf{4 2}}$. Mais la valeur exacte du signe fut découverte par G.F. Gamurrini en I87I, ce qui fut immédiatement admis par Fabretti ${ }^{43}$ : dans les mêmes tombes familiales, des gentilices comme Murina, Fremne, Amசia, Marcna, Lampe étaient écrits tantôt avec la lettre M sous la forme qu'elle a normalement en étrusque (c'est-à-dire trois traits verticaux reliés par des segments obliques), tantôt avec ce signe en forme de lambda grec; en outre dans certaines de ces inscriptions, comme celle du lampadaire de Cortone, on ne voyait pas de lettres M du type normal en étrusque mais certains mots présentaient des lettres L ayant leur tracé habituel, ce qui excluait que le signe $\Lambda$ y ait la valeur [1]. Et, si dans le CII, antérieur à cette découverte, Fabretti n'avait pas pu en tenir compte, il le fit dans le premier supplément qui parut en I872, où par deux fois il affirma sa pleine adhésion à la lecture de Gamurrini ${ }^{44}$. Soit que Casati n'ait pas tenu compte de ce changement d'attitude de l'auteur du CII, soit - ce qui paraît plus probable - il l'ait ignoré, on le voit rester fidèle encore en ıgı́ à la doctrine ancienne, dont la fausseté avait pourtant été démontrée plus de quarante ans auparavant.

Cet exemple montre bien que Casati n'était pas un véritable savant. Alors que Fabretti, partant de l'analyse raisonnée des documents à partir de la nouvelle hypothèse avancée par Gamurrini, avait abandonné sa position originelle, lui ne remit jamais en cause ce qu'il avait écrit dans sa publication de i873. Il s'en était tenu aux idées de sa jeunesse et n'avait pas suivi l'évolution des connaissances. Il est un peu pitoyable de le voir citer en I9ı́ comme seules autorités dans le domaine des études étrusques «mes anciens amis le sénateur Fabretti, le comte Conestabile

42 Glossarium Italicum, p. Io8o. Fabretti faisait état au début de sa notice de la lecture lumni (mais en ajoutant un point d'interrogation) et de l'interprétation par lumen (fortasse lumen apud Etruscos), mais signalait également la lecture avec M comme signe de sifflante (alii legunt luśni = Losna $i$. e. luna), avancée par Vermiglioli et Cavedoni.

43 Gamurrini I87ı, p.ı63-ı64, n. I (où l'auteur faisait déjà état de l'approbation “del mio amico Ariodante Fabretti»). L'auteur reprit la question à la suite de la parution du mémoire de C. Casati ( Non sarei tornato a parlare sul valore della lettera, che s’incontra in alcune iscrizioni etrusche, e che fu da me stabilita per la prima volta equivalere alla M, se non mi fosse pervenuta una memoria letta da M.C. Casati alla società letteraria di Lilla, in cui pone nuovamente in dubbio la sua lezione») pour réaffimer la justesse de sa lecture de ce $\Lambda$ comme $[\mathrm{m}]$ dans Gamurrini i873. Sur la question, voir maintenant Heurgon ig65 (= Heurgon ig86, p. I87-201).

44 CII, Supplément I, I872, p.2I, I9o. Dans les deux passages Fabretti cite le lampadaire de Cortone comme un des documents pour lesquels la nouvelle lecture du signe $\Lambda$ s’impose. À la p.ıgo, n. I, il cite Casati comme représentant de la thèse ancienne («il signor Casati in una Note sur la lettre $\Lambda$ dans l'alphabet étrusque»). 
et le Père Garrucci ${ }^{45}$ ». Tous étaient alors morts depuis longtemps: Ariodante Fabretti, né en ı8ı6, en ı894, Giancarlo Conestabile, né en ı824, en I877, Rafaelle Garrucci, né en i8II, en i885. Et il est frappant que Casati ne cite que des Italiens, alors que depuis un certain temps c'était plutôt du côté de l'Allemagne qu'il fallait chercher des représentants de l'état le plus récent de l'étruscologie ${ }^{46}$. En Igi4, cela faisait soixante-neuf ans qu'était paru le livre révolutionnaire de W.Deecke (I83I-I897), où, prenant le contrepied de la thèse que venait de soutenir W. Corssen mais qui ne faisait que reprendre la communis opinio des savants de l'époque, il avait démontré que l'étrusque n'avait rien à voir avec les langues italiques ${ }^{47}$. Casati menait donc ce qui était depuis longtemps un combat d'arrière-garde quand, en conclusion de son opuscule de I9ı4, il écrivait: “L'étude de ces inscriptions m’a amené à voir dans la langue étrusque une langue sœur de la langue latine, c'est-àdire ni finnoise, ni hongroise ${ }^{48}$, mais une langue italique. » Il faut dire à la décharge de Casati que, lorsqu'il s'était lancé dans le domaine des études étrusques, l'idée d'en faire une langue italique n'apparaissait en rien scandaleuse et était la doctrine la plus répandue. Et on aurait mauvais gré de reprocher à cet amateur que restait Casati de s'être laissé convaincre par la vision alors dominante qui faisait de cette langue un parler italique, proche du latin ou de l'ombrien. Simplement il ne sut pas changer d'idée par la suite.

Charles Casati n'a certainement pas marqué l'histoire de l'étruscologie. Collectionnant les antiquités étrusques, affichant des prétentions scientifiques qui le poussèrent à multiplier les interventions à l'Académie des inscriptions et belles-lettres et peuvent nous paraître ridicules aujourd'hui, cet ancien élève

45 Casati ıgı́a, p. XVII; le nom de Garrucci est écrit Garuci.

46 Une remarque critique sur la science allemande transparaît dans la suite de la phrase: "ces quatre mille mots étrusques que nous connaissons et que connaissaient bien [Fabretti, Conestabile, Garrucci] suffisent pour déterminer le caractère de la langue étrusque, mais ils ne sont pas assez connus par les autres personnes qui s'occupent de la langue étrusque, soit en Allemagne, soit en France, soit même en Italie, où cependant ces inscriptions étrusques sont étalées sous les yeux.»

47 Deecke i875 (à propos de Corssen I874). Que Deecke ait changé d'avis ensuite et soit retourné à partir de i88I aux idées de Corssen (sur le personnage et sa vie, Kronasser I957) n'empêcha pas que la thèse faisant de l'étrusque une langue italique fut alors pratiquement abandonnée. Sur la question et ses incidences sur Pauli et Danielsson, Wikander 2003, p. I6-I7.

48 Cette précision vise à répondre à ce qui a motivé la rédaction de l'opuscule, la publication de l'ouvrage de Jules Martha (I853-I932), Martha I9I3, où l'auteur soutenait l'appartenance de l'étrusque au groupe des langues finno-ougriennes. Sur la question, Prost 20I5, Hadas-Lebel 2015. 
de l'École des Chartes qui fit une carrière de magistrat ne fut certainement pas un grand savant. Il n'en reste pas moins représentatif de la période où il vécut, période qui marqua un changement radical dans les études étrusques et notamment celles portant sur la langue, avec l'émergence d'une approche réellement scientifique, sortant enfin des comparaisons impressionistes et approximatives avec le grec, le latin ou les autres parlers italiques pour s'attacher à l'étude interne, la plus rigoureuse possible, des documents - et, si on veut, le passage de la méthode étymologique à la méthode combinatoire ${ }^{49}$. Il ne fut pas un chercheur de premier plan: il se borna à reprendre, sans originalité (mais souvent sur un ton péremptoire!), ce que d'autres avaient dit avant lui et par exemple, dans la période initiale de ses travaux sur les Étrusques, il s'appuya sur l'autorité d'un personnage d'une tout autre stature scientifique et institutionnelle, Alfred Maury, qui s'était lui aussi intéressé aux Étrusques ${ }^{50}$. En fait, si on compare ce que ce professeur au Collège de France et directeur-général des Archives avait dit de la langue étrusque dans des mémoires présentés à l'Académie en i857 et I $858^{51}$ aux travaux de Casati, on ne note pas de différence fondamentale dans les principes de méthode suivis, toujours fondés sur des assonnances et des rapprochements assez vagues avec des mots de langues connues. Mais, si ce que Casati avait pu alors écrire n'était pas réellement en porte-à-faux par rapport à ce qu'était l'étruscologie de son temps, le progrès de la discipline allait rapidement le marginaliser - et réduire ses travaux à n'être plus que l'écho attardé d'un stade de la recherche désormais complètement dépassé.

\author{
Dominique Briquel \\ Archéologie et philologie d'Orient \\ et d'Occident umR 8546 \\ École Normale Supérieure \\ 45 rue d'Ulm \\ F-75230 Paris Cedex 05 \\ dominique.briquel@ens.fr \\ dominique.briquel@gmail.com
}

\footnotetext{
49 Sur les différentes méthodes d'approche de la langue étrusque, on pourra se reporter à Pallottino 1978.

50 Biographie de A. Maury dans Wallon ı894; sur le personnage et sa place dans l'histoire des sciences, Carroy, Richard 2007.

51 Maury I857, Maury I858.
} 


\section{Bibliographie}

Bonamici 1994: Marisa Bonamici, Orvieto. La necropoli di Cannicella, scavi della Fondazione per il Museo “Claudio Faina», Rome, I994.

Briguet 2002: Marie-Françoise Briguet, Les Urnes cinéraires étrusques de l'époque hellénistique, Musée du Louvre, Département des Antiquités Étrusques et Romaines, Paris, 2002.

Briquel 2013: Dominique Briquel, «Le voyage de O. A. Danielsson à Paris en I9o9 et le fonds épigraphique étrusque du musée du Louvre », Comptes Rendus de l'Académie des inscriptions et belles-lettres, 2013, p.375-395.

Carroy, Richard 2007: Jacqueline Carroy, Nathalie Richard (sous la direction de), Alfred Maury érudit et rêveur. Les sciences de l'homme au milieu du xIXe siècle, Rennes, 2007.

Casati i873: Charles Casati, La lettre $\Lambda$ dans l'alphabet étrusque, Paris, I873.

Casati I886: Charles Casati, intervention à la séance de l'Académie des inscriptions et belles-lettres, 5 février i886, Revue critique d'histoire et de littérature, 3o, I886, p. I40.

Casati I9ıа: Charles Casati, Les Étrusques. Leur langue et leur civilisation, Première livraison, La langue étrusque à l’Académie des inscriptions et à la Société des antiquaires de France, Paris, I9ı.

Casati I9ı/b : Charles Casati,Les Étrusques. Leur langue et leur civilisation, deuxième livraison, La civilisation étrusque, Paris, I9I4.

Corssen 1874: Wilhelm Corssen, Über die Sprache der Etrusker, Leipzig, I874.

Deecke I875: Wilhelm Deecke, Corssen und die Sprache der Etrusker. Eine Kritik, Stuttgart, 1875 .

Deecke ı877: Wilhelm Deecke, “Die etruskischen Zahlwörter », Adalbert Bezzenberger Beiträge zur Kunde der indogermanischen Sprachen, I, I877, p.96-ı16.

Fontaine I995: Paul Fontaine, “À propos des inscriptions śüina sur la vaisselle métallique étrusque », Revue des Études Anciennes, 97, I995, p. 20 I-2I6.

Gamurrini ı87: Gian Francesco Gamurrini, “Alfabeti etruschi di Chiusi », Annali dell'Instituto di Corrispondenza Archeologica, 43, I87I, p. I56-ı66.

Gamurrini I873: Gian Francesco Gamurrini, "Sul valore della lettera $\Lambda$ etrusca», Bullettino dell'Instituto di Corrispondenza Archeologica, 45, I873, p. 25o-252.

Gamurrini I880: Gian Francesco Gamurrini, Appendice al Corpus Inscriptionum Italicarum ed ai suoi Supplementi di Ariodante Fabretti, Florence, , 880.

Hadas-Lebel 20I5: Jean Hadas-Lebel, “Jules Martha et la controverse sur l'origine de l'étrusque ", dans Pour une histoire de l'archéologie, xvII 'eiècle-I945. Hommage de ses collègues et amis à Eve Gran-Aymerich, sous la direction d'Annick Fennet et Natacha Lubtchansky, Scripta Receptoria 5, Bordeaux, 2015, p. 273-28o. 
Helbig 188I: Wolfgang Helbig, "Orvieto», Bullettino dell'Instituto di Corrispondenza Archeologica, 53, I88I, p. 26I-282.

Heurgon ig65: Jacques Heurgon, “Note sur la lettre $\Lambda$ dans les inscriptions étrusques », dans Studi in onore di Luisa Banti, Rome, I965, p. I77-I89.

Heurgon ig86: Jacques Heurgon, Scripta Varia, collection Latomus igi, Bruxelles, I986.

Kaimio 2010: Jorma Kaimio, The Cippus Inscriptions of the Museo Nazionale di Tarquinia, Materiali del Museo Archeologico Nazionale di Tarquinia $\mathrm{7} 7$, Rome, 2010.

Klakowicz 1974: Beatrix Klakowicz, La necropoli anulare di Orvieto, II, Cannicella e terreni limitrofi, Rome, 1974 .

Körte 1877: Gustav Körte, “Sulla necropoli di Orvieto», Annali dell'Instituto di Corrispondenza Archeologica, 49, I877, p. 95-II6.

Kronasser 1957: Heinz Kronasser, «Deecke, Wilhelm », dans Neue deutsche Biographie, III, Berlin, I957, p.554-555.

Lanzi I789: Luigi Lanzi, Saggio di lingua etrusca e di altre antiche d'Italia per servire alla storia de' popoli, delle lingue e delle belle arti, Rome, 1789 .

Lazzarini 1994: Andrea Lazzarini, «Documenti storici sulla Fabbrica del Duomo di Orvieto (II54-I864)", dans Saggi inediti. Per la storia della politica italiana e della città di Orvieto, Rome, I994, p. 123-136.

Mancini ı885: Riccardo Mancini, “Orvieto », Notizie degli Scavi, 1885, p. I5-17.

Mancini I888: Riccardo Mancini, “VI. Orvieto », Notizie degli Scavi, p.348-349.

Martha Igi3: Jules Martha, La langue étrusque. Affinités ougro-finnoises, précis grammatical, textes traduits et commentés, dictionnaire étymologique, Paris, I9I3.

Maury i857: Alfred Maury, "Système alphabétique et vocalisation de la langue étrusque ( $\mathrm{I}^{\mathrm{er}}$ mémoire)", Comptes Rendus de l'Académie des inscriptions et belles-lettres, 1857 , p. 96-97.

Maury I858: “Mémoire sur la langue étrusque (2 $2^{\mathrm{e}}$ mémoire)», Comptes Rendus de l’Académie des inscriptions et belles-lettres, I858, p. I66-179.

Pallottino 1978: La Langue étrusque. Problèmes et perspectives, traduit par J. Heurgon, Monographies linguistiques 3, Paris, 1978.

Prost 20I5: Célia Prost, “La réception critique de La Langue étrusque de Jules Martha: l'apport des archives privées", dans La Construction de l'étruscologie au début du $X x^{e}$ siècle, sous la direction de Marie-Laurence Haack avec la collaboration de Martin Miller, Ausonius Éditions, Scripta Receptoria 3, Bordeaux, 20I5, p.67-9I.

Rix I963: Helmut Rix, Das etruskische Cognomen. Untersuchungen zu System, Morphologie und Verwendung der Personennamen auf den jüngeren Inschriften Nordetruriens, Wiesbaden, ig63. 
Sclafani 20I0: Marina Sclafani, Urne fittili chiusine e perugine di età medio e tardo ellenistico, Archaeologica i6o, Tyrrhenica 7, Rome, 20 o.

Wallon i894: Henri Wallon Henri, “Notice sur la vie et les travaux de M. LouisFerdinand-Alfred Maury, membre de l'Académie des Inscriptions et BellesLettres ", Comptes Rendus de l'Académie des inscriptions et belles-lettres, I894, p.53o-579.

Wikander 2003: Charlotte Wikander, Örjan Wikander, Etruscan Inscriptions from the Collections of Olof August Danielsson: addenda to CIE II, I, 4, Medelhavsmuseet Memoir ı, Stockholm, 2003. 